\title{
Lactobacillus acidipiscis Induced Regulatory Gamma Delta T Cells and Attenuated Experimental Autoimmune Encephalomyelitis
}

\author{
Saisai Ren ${ }^{2 t}$, Xiaorong Zhang ${ }^{1+}$, Hongbing Guan ${ }^{1 *}$, Lihong $W^{1}{ }^{1}$, Miao $\mathrm{Yu}^{1}$, Dan Hou ${ }^{1}$, \\ Yongyong Yan ${ }^{1}$ and Xuechun Fang ${ }^{2}$ \\ ${ }^{1}$ Guangzhou Key Laboratory of Basic and Applied Research of Oral Regenerative Medicine, Department of Basic Science of \\ Stomatology, Affiliated Stomatology Hospital of Guangzhou Medical University, Guangzhou, China, ${ }^{2}$ Guangzhou Medical \\ University, Guangzhou, China
}

OPEN ACCESS

Edited by:

Lloyd Kasper,

Dartmouth College, United States

Reviewed by:

lain Comerford,

University of Adelaide, Australia

Cristina Ulivieri,

University of Siena, Italy

*Correspondence:

Hongbing Guan

hongbing2015@qq.com

${ }^{\dagger}$ These authors have contributed equally to this work

Specialty section:

This article was submitted to

Multiple Sclerosis and

Neuroimmunology,

a section of the journal

Frontiers in Immunology

Received: 30 October 2020

Accepted: 27 January 2021

Published: 19 February 2021

Citation:

Ren S, Zhang X, Guan H, Wu L, Yu M,

Hou D, Yan Y and Fang X (2021)

Lactobacillus acidipiscis Induced Regulatory Gamma Delta T Cells and Attenuated Experimental Autoimmune Encephalomyelitis.

Front. Immunol. 12:623451. doi: 10.3389/fimmu.2021.623451
Multiple sclerosis is a chronic autoimmune disease involving the central nervous system, and shows a high disability rate. Its pathogenesis is complicated, and there is no good treatment. In recent years, with in-depth studies on the regulation of gastrointestinal flora, the relationship between the mammalian immune system and the intestinal flora has been extensively explored. Changes in the composition and structure of the gastrointestinal flora can affect the characteristics and development of the host immune system and even induce a series of central nervous system inflammation events. The occurrence and development of multiple sclerosis are closely related to the continuous destruction of the intestinal barrier caused by intestinal dysbacteriosis. In this study, we analyzed Lactobacillus acidipiscis in a mouse model of experimental autoimmune encephalomyelitis (EAE). We found that the amount of $L$. acidipiscis in the intestinal tract was inversely proportional to the progress of EAE development. In addition, the number of $\mathrm{CD}^{+}{ }^{+} \mathrm{FOXP3}^{+}$regulatory $\mathrm{T}$ cells in the mesenteric lymph nodes of mice increased significantly after the mice were fed with $L$. acidipiscis, and the differentiation of CD4 ${ }^{+} T$ cells to Th1 and Th17 cells was inhibited. However, the protective effect of $L$. acidipiscis was lost in $\gamma \delta$ T cell-deficient mice and hence was concluded to depend on the presence of regulatory $\gamma \delta T$ cells in the intestinal epithelium. Moreover, including $L$. acidipiscis enhanced the development of $V_{\gamma} 1^{+} \gamma \delta T$ cells but suppressed that of $V_{\gamma} 4^{+} \gamma \delta T$ cells. In summary, our results demonstrated the ability of $L$. acidipiscis to induce generation of regulatory $\gamma \delta$ T cells that suppress the development of the encephalomyelitic Th1 and Th17 cells and the progress of EAE.

Keywords: Lactobacillus acidipiscis, $\gamma \delta \mathrm{T}$ cells, regulatory T cells, T helper cells, multiple sclerosis, experimental autoimmune encephalomyelitis

\section{INTRODUCTION}

More than 2.5 million people worldwide suffer from multiple sclerosis (MS), which is a chronic autoimmune disease affecting the central nervous system (CNS) and mainly occurring in young women with a high disability rate $(1,2)$. The pathogenesis of MS is very complex and its exact mechanism is unclear. Inflammation of the CNS and demyelination of the nerves are 
the main signs of MS. At present, MS is generally believed to be caused by autoreactive immune cells infiltrating the blood-brain barrier $(\mathrm{BBB})$ with abnormal responses to autoantigens of the CNS, and myelin-specific $\mathrm{CD} 4{ }^{+} \mathrm{T}$ cells are key to the occurrence of this disease (3). $\mathrm{CD}^{+}$helper $\mathrm{T}$ (Th) cells are considered to play the most important role in the pathogenesis of MS. Th1 and Th17 cells promote inflammation of the CNS, while Th2 and regulatory $\mathrm{T}$ (Treg) cells inhibit it. Experimental autoimmune encephalomyelitis (EAE) is a disease model mediated by specific sensitized $\mathrm{CD}^{+} \mathrm{T}$ cells, and is constructed by immunizing experimental animals with myelin sheath protein. This model is consistent with the induction method of MS, specifically combining myelin oligodendrocyte glycoprotein residues 35-55 $\left(\mathrm{MOG}_{35-55}\right)$ with immunostimulant to induce the generation of pathogenic Th1 and Th17 cells (4).

Changes of intestinal microflora may lead to maladjustments of the immune response in the intestinal tract and other peripheral lymphoid sites including the CNS. There is increasing evidence that signal communication on the microbe-gut-brain response axis is closely related to the occurrence of MS, Parkinson's disease, Alzheimer's disease, depression, and other CNS diseases (5). Intestinal symbiotic bacteria that induce the generation of $\mathrm{CD}^{+} \mathrm{T}$ cells have been shown to change the severity of the demyelination of the CNS, and changing some bacterial groups in the intestinal tract could lead to a proinflammatory state, which in turn may lead to the development of autoimmune diseases, especially MS. Oral treatment of mice with antibiotics has been shown to reduce the severity of EAE by reducing inflammation and increasing accumulation of FOXP3 in mesenteric and cervical lymph nodes (6). Our results in 2016 demonstrated that the attenuation of EAE seen following CD44 gene deletion in mice, i.e., in CD44 knockout $(\mathrm{CD} 44 \mathrm{KO})$ mice, may result from alterations in the gut microbiota and short-chain fatty acids (SCFAs). Furthermore, our studies also demonstrated that phenotypes of gene knock-out animals in general may be shaped by gut microbiota (7).

Lactobacillus acidipiscis is an aerobic gram-positive lactobacillus acidophilus, and was recently discovered to be present in the lungs of healthy people, with this presence related to the function of pulmonary $\gamma \delta \mathrm{T}$ cells (8). In the intestinal epithelial lymphocytes (IELs) of mice, specifically in the duodenum and jejunum, the percentage of T cells consisting of $\gamma \delta \mathrm{T}$ cells was observed to be as high as $70 \%$, much higher than that of $\alpha \beta \mathrm{T}$ cells (9). More interestingly, $\gamma \delta \mathrm{T}$ cells constitute the main target of intestinal bacteria. Aerobic bacteria or facultative anaerobes can activate $\gamma \delta$ T cells more effectively than can anaerobes. One of the effectors produced by $\gamma \delta \mathrm{T}$ cells has been found to be IL-17, and hence these T cells are called IL-17-producing $\gamma \delta \mathrm{T}(\gamma \delta 17 \mathrm{~T})$ cells. In EAE, $\gamma \delta 17 \mathrm{~T}$ cells have shown both positive and negative effects, namely on the one hand aggravating the disease by increasing the quantity of Th 17 cells, but also on the other hand alleviating the disease by promoting apoptosis of Th17 cells and enhancing the function of Treg cells. The positive and negative effects of $\gamma \delta 17 \mathrm{~T}$ cells may be related to the heterogeneity of $\gamma \delta \mathrm{T}$ cells. The expression of CCR5 in ${\mathrm{V} \gamma 1^{+}}^{+} \delta 17 \mathrm{~T}$ cells can enhance the function of Treg cells and induce the apoptosis of $\alpha \beta \mathrm{T}$ cells through FAS-FASL signal transduction. However, the expression of CCR6 in $\mathrm{V} \gamma 4^{+}$ $\gamma \delta 17 \mathrm{~T}$ cells promotes the generation of pathological Th17 cells $(10,11)$. Therefore, inducing protective regulatory $\gamma \delta \mathrm{T}$ cells has become a new strategy for treating MS. Studies have shown that intestinal bacteria can stimulate macrophages and dendritic cells to produce IL- 1 and IL-23, and activate $\gamma \delta 17 \mathrm{~T}$ cells via the guanosine exchange factor 1 (VAV1) signaling pathway. According to the composition of $\mathrm{T}$ cell receptors, the peripheral $\gamma \delta \mathrm{T}$ cells in mice can be divided into two main subsets: $\mathrm{V} \gamma 1^{+}$ and $\mathrm{V} \gamma 4^{+}(12)$. However, $\mathrm{V} \gamma 1^{+} \gamma \delta \mathrm{T}$ cells have been shown to produce more Th2-type cytokines such as IL-4, while $\mathrm{V} \gamma 4^{+} \gamma \delta$ $\mathrm{T}$ cells have been shown to preferentially produce IL-17A. In an experiment involving PMA/ionomycin-activated $\mathrm{V} \gamma 1^{+}$and $\mathrm{V} \gamma 4^{+} \gamma \delta \mathrm{T}$ cells, 20 differentially expressed genes were identified in the two cell subtypes, with most of these genes related to cytokines, cell differentiation, transcription and translation (13). In addition, intestinal probiotics were shown to produce protective short-chain fatty acids (SCFAs) such as acetate, propionate and butyrate acids (14), which have been shown to regulate the immune balance of intestinal and extraintestinal lymphoid tissues and organs, induce the differentiation of $\mathrm{CD} 4{ }^{+}$ $\mathrm{T}$ cells from Th1/Th17 cells to Th2/Treg cells, and induce the production of regulatory dendritic cells (DCs) and migration of Treg cells to gut-related lymphoid tissues (GALTs).

In rodent models, discrepancies in gut microbiota were found to be associated with in some cases susceptibility to EAE and other cases resistance to EAE (15). Previous studies from our laboratory showed that CD44 may also regulate inflammation, in as much as CD44 deficiency inhibits proinflammatory Th1 and Th17 cells while promoting $\mathrm{CD} 4^{+}$Th2 and Treg cell differentiation (16). In fact, CD44 deficiency led to decreased inflammation and amelioration of an experimental form of EAE. In those studies, CD44 gene deletion led to the alteration of gut microbiome and attenuation of EAE with simultaneously increase of Treg as well as decrease of Th17 (7).

In our current study, we found that L. acidipiscis became significantly more abundant in the intestinal flora of the EAEresistant $(\mathrm{CD} 44 \mathrm{KO})$ mice, having become the predominant lactobacillus, and the number of $\gamma \delta \mathrm{T}$ cells in the intestinal tissue increased significantly. L. acidipiscis induced resistance of otherwise EAE-susceptible mice to EAE by inducing the proliferation of protective Treg cells and inhibiting the differentiation to Th1 and Th17 cells. In addition, L. acidipiscis was shown to be able to induce the generation of $\mathrm{V} \gamma 1^{+} \gamma \delta \mathrm{T}$ cells having inhibitory effects on MS, with these T cells denoted as regulatory $\gamma \delta \mathrm{T}$ cells. In the absence of these regulatory $\gamma \delta \mathrm{T}$ cells, L. acidipiscis did not protect mice from EAE. Neither a clinical correlation between L. acidipiscis and MS nor its application in the clinical treatment of MS was investigated in the current work, but the experimental results that were obtained should provide the basis for further research in this field.

\section{MATERIALS AND METHODS}

\section{Mice and Reagents}

Specific-pathogen-free (SPF) C57BL/6 female mice that were 6-8 weeks old were purchased from Guangdong Medical 
Laboratory Animal Center. Also, SPF CD44KO female mice that were 6-8 weeks old were purchased from Jackson Laboratory and TCR $\delta^{-/-}$female mice that were 6-8 weeks old were purchased from Shanghai Model Organisms. All experimental animals were maintained under specific pathogen-free conditions at Guangzhou Medical University. All animal experiments were conducted under the protocols approved by and in accordance with the guidelines of the Institute Animal Care and Use Committee of the University. Myelin oligodendrocyte glycoprotein $\left(\mathrm{MOG}_{35-55}\right)$ peptide was purchased from GL Biochem (Shanghai, China). Pertussis toxin was purchased from Tocris. Incomplete Freund's adjuvant was purchased from Sigma. Antibodies and isotypes were purchased from eBioscience and BioLegend. Cell stimulation cocktail (plus protein transport inhibitors, $500 \times$ ) was purchased from eBioscience.

\section{Microbial Analysis in the Intestinal and Lactobacillus acidipiscis Culture}

Fecal samples of EAE mice, specifically of CD44KO (KO) mice and C57BL/6 wild-type (WT) mice, were collected according to the procedure described previously (17). The microbial community of mouse intestinal contents was further analyzed using $16 \mathrm{~S}$ rDNA. 16S rRNA amplicons were generated for the V3-V4 hypervariable regions of the fecal samples. Moreover, the Lactobacillus acidipiscis 10851 strain and Escherichia coli (E. coli) used in this study were obtained from the China Center of Industrial Culture Collection (CICC, Beijing, China). The strains were amplified in MRS liquid bacterial medium at $37^{\circ} \mathrm{C}$ for $24 \mathrm{~h}$, and L. acidipiscis 10851 or E. coli solution was obtained. The solution was subjected to centrifugation at $3,000 \mathrm{~g}$ for $5 \mathrm{~min}$, and the resulting supernatant was discarded to obtain the L. acidipiscis 10851 or E. coli precipitate. Each precipitate was suspended in sterile phosphate-buffered saline (PBS, pH 7.4), and subjected to another centrifugation at 3,000 g for $5 \mathrm{~min}$. Then, the resulting supernatant was discarded, and the number of bacteria in the remaining liquid was counted after repeated washing. Final bacterial dilutions were carried out according to bacterial colony forming units $(\mathrm{cfu} / \mathrm{mL})$ at the indicated bacterial cell densities in different buffers or media.

\section{Collection of Feces and Analysis of SCFAs}

The fecal contents were collected from C57BL/6 mice fed with E. coli or $L$. acidipiscis before being subjected to $\mathrm{MOG}_{35-55}$ peptide immunization. Feces were collected on day 15 of the immunization as described previously (18). Fecal contents (100 mg) were acidified with 25\% metaphosphoric acid for $30 \mathrm{~min}$ on ice and then centrifuged at $12,000 \mathrm{~g}$ for $15 \mathrm{~min}$ at $4^{\circ} \mathrm{C}$. Supernatants were filtered using Ultra free MC columns (0.22 $\mu \mathrm{m}$ GVDurapore, ThermoFisher Scientific) at $12,000 \mathrm{~g}$ for $4 \mathrm{~min}$ at $4{ }^{\circ} \mathrm{C}$. And then the eluates were analyzed using a GC-FID instrument.

\section{Probiotic Protection and EAE Induction}

C57BL/6 mice (20 animals) were randomly divided into four groups: C57BL/6 mice that received fecal transfer from C57BL/6 mice, those from CD44 KO mice, and E. coli and L. acidipiscis protection groups (using 10851 strain). Moreover, TCR $\delta^{-/-}$ mice (10 animals) were randomly assigned to two groups: E. coli and $L$. acidipiscis protection groups.

Before carrying out the fecal transfers, recipient mice were treated with streptomycin and ampicillin to deplete endogenous gut microbiota. As described previously (19), all mice were given antibiotics (penicillin and streptomycin, $1 \mathrm{mg} / \mathrm{mL}$ aqueous solution, $100 \mu \mathrm{l}$ ) by carrying out oral gavage for two consecutive days. Twenty-four hours after the second antibiotic feeding, C57BL/6 mice were fed fecal solution $(200 \mu \mathrm{l})$ collected from $\mathrm{C} 57 \mathrm{BL} / 6$ or $\mathrm{CD} 44 \mathrm{KO}$ mice for two consecutive days. Using a procedure similar to that used for the fecal transfer groups, some of the mice were given E. coli and others L. acidipiscis $\left(6 \times 10^{8}\right.$ $\mathrm{cfu} / \mathrm{mL}, 200 \mu \mathrm{l})$ using oral gavage for two consecutive days. At the same time, mice deficient in $\gamma \delta$ T cells (TCR $\delta^{-/-}$) were fed E. coli or L. acidipiscis 10851 in the same manner.

EAE was observed to be induced in all mice by immunizing them with $150 \mu \mathrm{g}$ of $\mathrm{MOG}_{35-55}$ in CFA-containing heatkilled Mycobacterium tuberculosis (strain H37Ra, $6 \mathrm{mg} / \mathrm{mL}$ ) as described previously (20). Then, on days 0 and 2 postimmunization, the mice were treated intraperitoneally with, respectively, 200 and $400 \mathrm{ng}$ of pertussis toxin. Mice were analyzed every day, and severity of EAE was scored using the 0-5 scoring grade: 0 , asymptomatic; 1 , tail tension loss; 2 , unilateral hind limb paralysis; 3, paralysis of hind legs on both sides; 4, paralysis of forelimb; and 5, moribund.

\section{Detection of Th Subsets in vivo After $L$. acidipiscis Feeding}

On the 15th day after the C57BL/6 mice were immunized with the $\mathrm{MOG}_{35-55}$ peptide, cells were isolated from the mesenteric lymph nodes of C57BL/6 mice after they were fed E. coli or L. acidipiscis. To investigated the frequency of Treg cells in the mesenteric lymph nodes, cells were stained with FITCconjugated anti-mouse-CD4 (GK1. 5, eBioscience) and APCconjugated anti-mouse FOXP3 antibody (FJK-16s, eBioscience). In addition, part of the cells isolated from the mesenteric lymph nodes were re-stimulated with $\mathrm{MOG}_{35-55}$ in vitro to observe the differentiation of Th1 and Th17. These encephalitogenic $\mathrm{CD}^{+} \mathrm{T}$ cells were cultured in RPMI1640 medium (Gibco BRL) with $10 \%$ FCS and $\mathrm{MOG}_{35-55}(30 \mu \mathrm{g} / \mathrm{mL})$. Twentyfour hours later, the cells were collected and stained with FITC-conjugated anti-mouse-CD4 (GK1. 5, eBioscience), PEconjugated anti-mouse IL-17A antibody (eBio17B7, eBioscience) and PerCP-conjugated anti-mouse IFN- $\gamma$ antibody (XMG1. 2 , eBioscience). The concentrations of IFN- $\gamma$, IL-17A, IL10 and IL-13 in the supernatant were determined by using a commercial ELISA kit.

\section{$\gamma \delta \mathrm{T}$ Activation and CD4 ${ }^{+} \mathrm{T}$ Differentiation in vitro With $L$. acidipiscis Stimulation}

For $\gamma \delta \mathrm{T}$ activation, flat-bottom 12-well plates were coated with $500 \mu \mathrm{l}$ of purified anti-mouse TCR $\gamma / \delta$ antibody (UC7-13D5, $1 \mu \mathrm{g} / \mathrm{ml}$, BioLegend) at $4^{\circ} \mathrm{C}$ overnight as described previously (21). Splenocytes were collected from EAE-C57BL/6 mice on 
day 15 post-immunization when EAE symptoms peaked, and $\mathrm{B}$ cells were removed from the population of splenocytes by carrying out magnetic separation using an EasySep FITC Selection Kit (Stemcell). The remaining cells were added to the Ab-coated wells and cultured in RPMI1640 medium (Gibco BRL) supplemented with $10 \%$ fetal calf serum and IL-2 $(200 \mathrm{IU} / \mathrm{ml})$ for 8 days. The resulting cells $\left(2 \times 10^{6}\right.$ cells $)$ were co-cultured with E. coli or L. acidipiscis $\left(1 \times 10^{7} \mathrm{cfu}\right)$ at a ratio of $5: 1$ splenocyte for 3 days. Cells were collected and stained with FITC-conjugated anti-mouse-TCR V $\gamma 1.1 / \mathrm{Cr} 4$ antibody (2.11, Biolegend), and APC-conjugated anti-mouse TCR V $\gamma 2$ antibody (UC3-10A6, Biolegend).

$\mathrm{CD}^{+} \mathrm{T}$ differentiation in vitro was performed as previously described with minor modification (16). Briefly, splenocytes were prepared from EAE-C57BL/6 mice on day 15 postimmunization, co-cultured with $E$. coli or $L$. acidipiscis (1 $\left.\times 10^{7} \mathrm{cfu}\right)$ at a ratio of $5: 1$ splenocyte and $30 \mu \mathrm{g} / \mathrm{ml}$ of $\mathrm{MOG}_{35-55}$ for $24 \mathrm{~h}$, followed by stimulation with Cell
Stimulation Cocktail (plus protein transport inhibitors, $500 \times$, eBioscience) for $5 \mathrm{~h}$. Production of IL-4 and IL-17A in the $\mathrm{CD} 4^{+} \mathrm{T}$ cells were then detected by intracellular staining and flow cytometry.

\section{Intracellular Staining, Flow Cytometry and Cytokine Assays}

For staining of intracellular IFN- $\gamma$, FOXP3, IL-4 and IL-17A, cells were stimulated for 5-6 h with Cell Stimulation Cocktail (plus protein transport inhibitors, $500 \times$, eBioscience), which is a cocktail of phorbol 12-myristate 13-acetate (PMA) and ionomycin. The cells were harvested, washed twice with PBS, and analyzed for the presence of Treg and Th17 cells. The cells were then fixed, permeabilized, and stained with IFN- $\gamma$-PerCP, FOXP3-APC, IL-4-PE-Cyanine7 (11B11, eBioscience) and IL$17 \mathrm{~A}-\mathrm{PE}$ antibodies. Fluorescence signals were detected using a BD FACS Canto II flow cytometer (BD Biosciences, San Jose, CA,

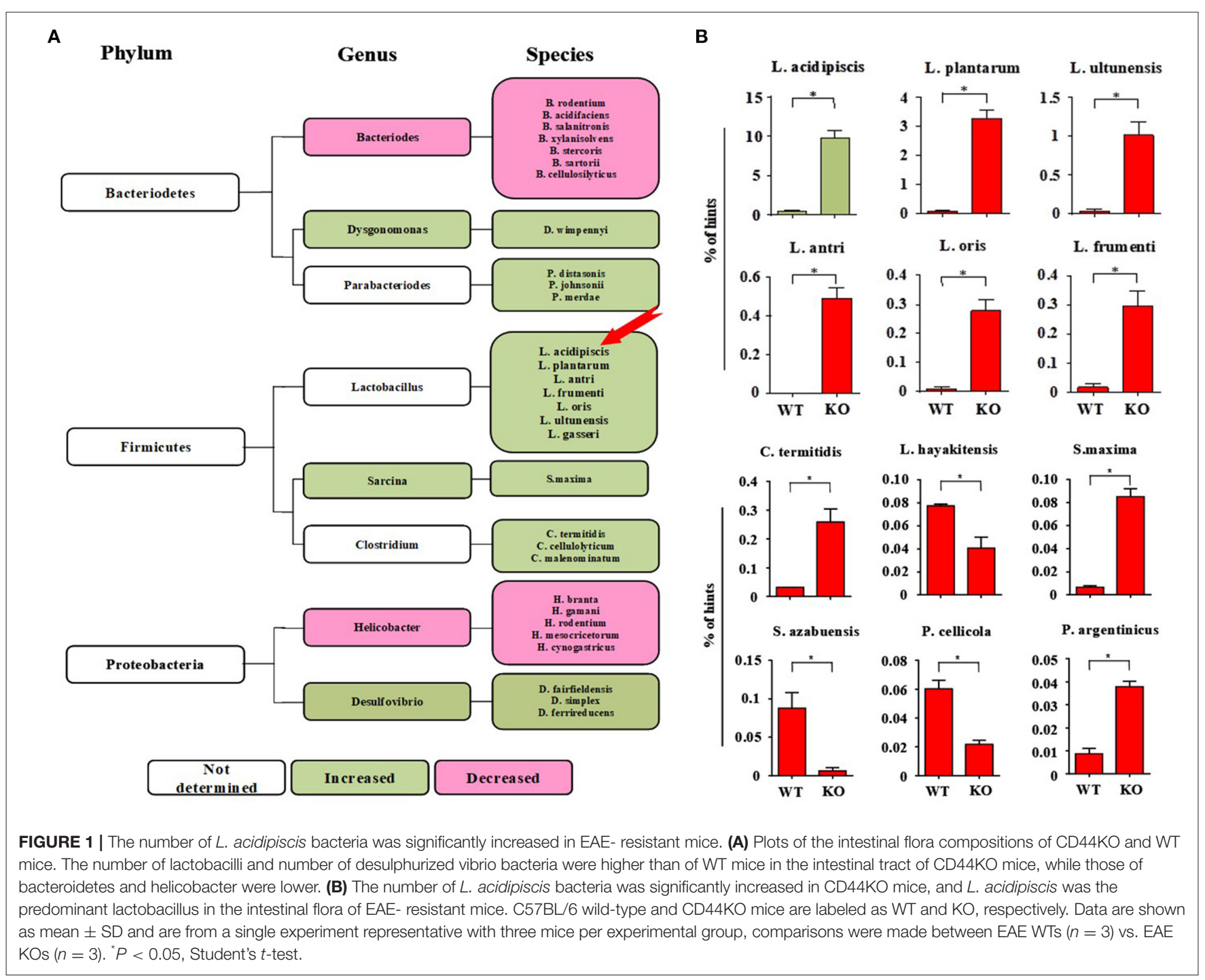


USA). Data were analyzed by using FlowJo (Tree Star, Ashland, OR, USA) software.

\section{Determination of Concentrations of Cytokines Using ELISA}

The concentrations of IFN- $\gamma$, IL-17A, IL-10 and IL-13 in supernatants were determined by using a commercial ELISA kit (BioLegend) according to the manufacturer's instructions.

\section{Histopathology}

Intestinal tissues were removed from mice as a result of subjecting heart to heparin-PBS perfusion and fixed in $10 \%$ paraformaldehyde. These intestinal tissues were then treated with GL3 antibody stain, and examined under an optical microscope in order to visualize $\gamma \delta$ T cells.

\section{Statistical Analysis}

Statistical difference between different groups was analyzed by performing the Student's $t$-test using Graph Pad Prism 6.2 software (GraphPad Software Inc, San Diego, CA, USA). The nonparametric data (EAE scoring) were analyzed using the Mann-Whitney $U$ test. Values of $P<0.05$ were considered to indicate statistical significance. The statistical analysis data are presented in the manuscript as mean $\pm \mathrm{SD}$ or mean \pm SEM.

\section{RESULTS}

\section{The Number of $L$. acidipiscis Bacteria Was Significantly Increased in EAE-Resistant Mice}

On the 15th day after mice were immunized with $\mathrm{MOG}_{35-55}$ peptide, 16s rRNA V4 sequencing analysis of the intestinal flora of the mice was performed. We found that the intestinal flora composition of CD44KO mice was significantly different from that of WT. The number of L. acidipiscis in the intestines of the CD44KO mice was much higher than that in C57BL/6 mice (Figure 1A) $(p<0.05)$. The part of the intestinal flora compositions of the EAE-resisitant and WT mice are shown in Figure 1B. Significantly different compositions of intestinal bacteria strains in the CD44KO and WT group were observed. Also, significantly more $L$. acidipiscis bacteria than other lactobacilli were found in the CD44KO group, and L. acidipiscis was the predominant lactobacillus in the intestinal flora of the CD44KO mice.

\section{L. acidipiscis Induced the Production of Intestinal SCFA}

To explore the effect of $L$. acidipiscis on the content of SCFAs in the intestinal tracts of C57BL/6 mice that had been fed L. acidipiscis, an EAE model was established by immunizing

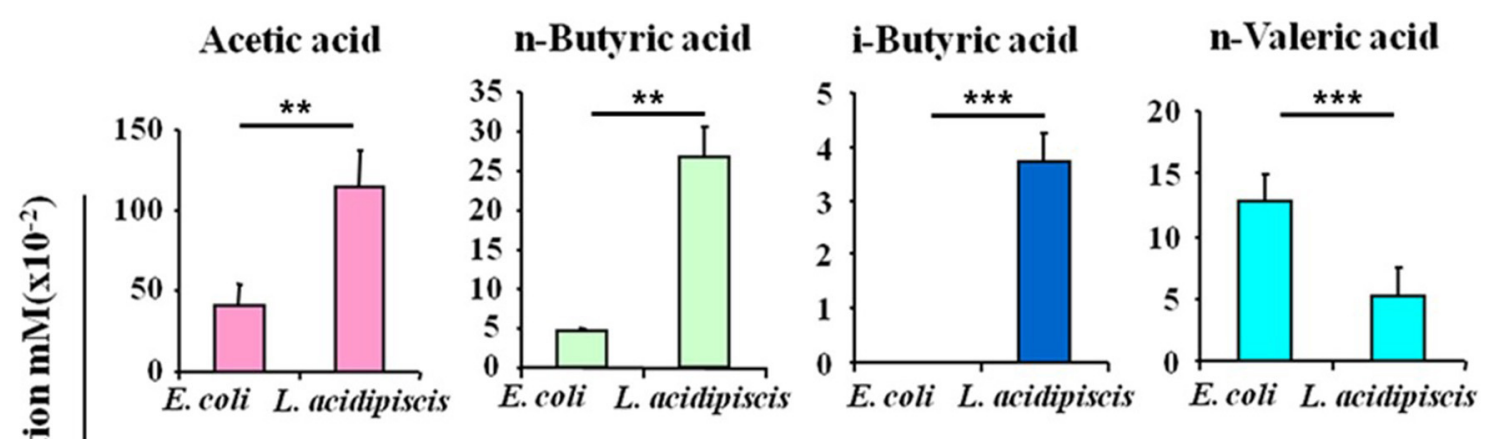

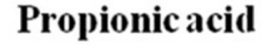

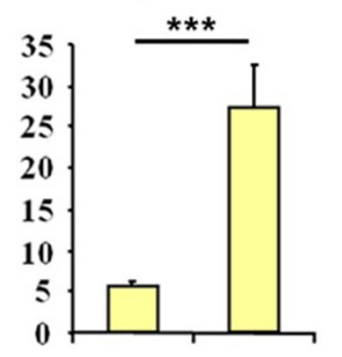

E. coli L. acidipiscis

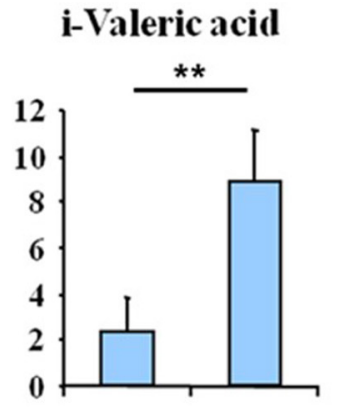

E. coli L. acidipiscis
n-Caproic acid

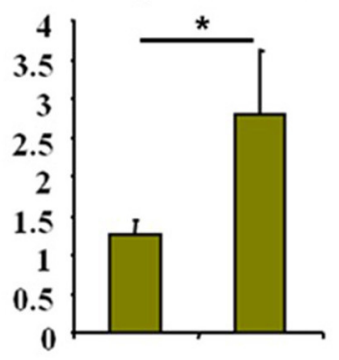

E. coli L. acidipiscis

FIGURE 2 | L. acidipiscis induced the production of intestinal SCFA. Concentrations of SCFAs in the fecal contents of EAE-WT mice that had been fed E. coli or $L$. acidipiscis. Data shown are mean $\pm \mathrm{SD}\left({ }^{\star} P<0.05,{ }^{\star \star} P<0.01\right.$, ${ }^{\star \star \star} P<0.001$, Student's $t$-test) and are from a single experiment representative with five mice per experimental group. 
some of the C57BL/6 mice with the $\mathrm{MOG}_{35-55}$ peptide, and treating other mice with $E$. coli as the control group. Mouse feces were collected on day 15 after the immunization with the $\mathrm{MOG}_{35-55}$ peptide, and the SCFA content in mouse feces was determined using the GC-FID method. The abundances and concentrations of acetic acid, n-butyric acid, i-butyric acid, nvaleric acid, propionic acid, i-valeric acid and $n$-caproic acid in each sample are shown in Figure 2. The amounts of acetic acid $(p<0.01)$, n-butyric acid $(p<0.01)$, i-butyric acid $(p<0.001)$, propionic acid $(p<0.001)$, i-valeric acid $(p<0.01)$ and $n-$ caproic acid $(p<0.05)$ in the feces of C57BL/6 mice fed with $L$. acidipiscis were significantly higher than for those fed with E. coli. Furthermore, the data showed a significant difference $(p<0.001)$ in the concentration of $n$-valeric acid between the EAE-WT mice that received $E$. coli and those receiving $L$. acidipiscis, indicating that $L$. acidipiscis could induce protective immunophenotypes by synthesizing acetic acid and other SFCAs in the intestinal tracts of EAE-susceptible mice.

\section{L. acidipiscis Induced Resistance to EAE in Susceptible Mice}

Seven days before being immunized with the $\mathrm{MOG}_{35-55}$ peptide, WT (Figures 3A,B) and $\mathrm{TCR} \delta^{-/-}$(Figure 3C) mice were inoculated with various materials: some mice were inoculated with fecal material of CD44KO mice (Figure 3A), others with $L$. acidipiscis (Figures 3B,C), and still others with the fecal material of WT mice (Figure 3A) or E. coli bacteria (Figures 3B,C), respectively, as controls. Feeding $\mathrm{CD} 44 \mathrm{KO}$ mouse feces or an $L$. acidipiscis bacteria suspension to WT mice significantly inhibited the occurrence of EAE and reduced the degree of disease (Figures 3A,B). However, the occurrence of EAE was not significantly inhibited when $L$. acidipiscis was fed to mice with a deficiency of $\gamma \delta \mathrm{T}$ cells $\left(\mathrm{TCR} \delta^{-/}\right.$) (Figure 3C), suggesting that the protective effect of $L$. acidipiscis on susceptible mice requires the presence of $\gamma \delta \mathrm{T}$ cells.

\section{L. acidipiscis Induced Treg Cell Development and Inhibited Pathological Th1 Cell and Th17 Cell Differentiation}

On the 15th day after mice were immunized with $\mathrm{MOG}_{35-55}$ peptide, the number of Treg cells from mesenteric lymph nodes of C57BL/6 mice that were fed L. acidipiscis was significantly greater than that for $\mathrm{C} 57 \mathrm{BL} / 6$ mice that were instead fed control material, and the differentiation of $\mathrm{CD} 4^{+} \mathrm{T}$ cells to Th1 and Th17 cells was inhibited. The production of protective IL-10 and that of IL-13 were each increased, while the production of pathological IFN- $\gamma$ and that of IL-17A were each significantly decreased, indicating that $L$. acidipiscis may regulate the differentiation of cerebrospinal inflammatory $\mathrm{CD} 4^{+} \mathrm{T}$ cells and induce a deviation of the protective $\mathrm{T}$ cell immune response (Figure 4).

\section{The Number of $\gamma \delta$ T Cells Increased Significantly in the Intestines of EAE- Resistance Mice}

We also set out to compare the numbers of $\gamma \delta$ T cells in EAEresistant mice and EAE-susceptible mice. For this purpose on
A

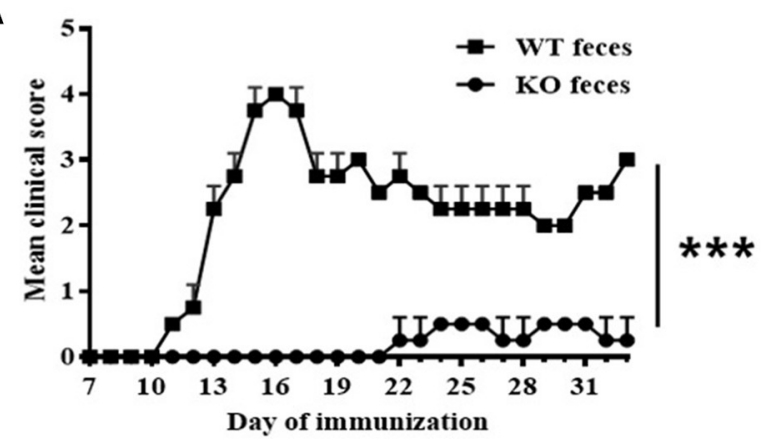

B

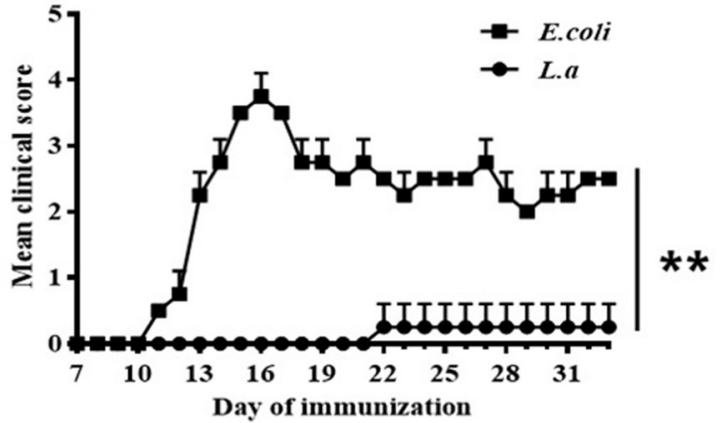

C

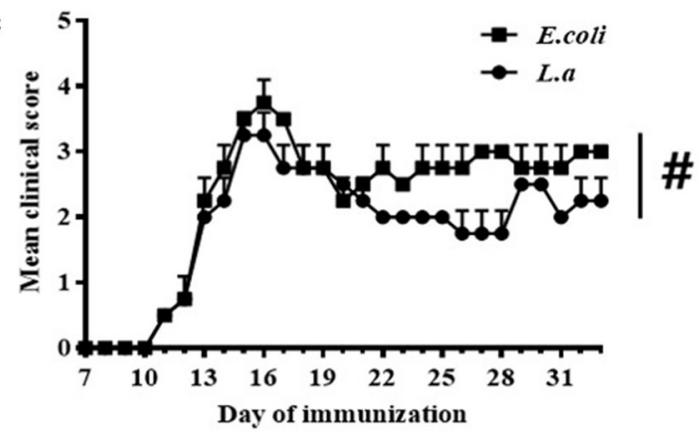

FIGURE 3 | L. acidipiscis induced resistance to EAE in susceptible mice. (A) Results showing feces of CD44KO mice having induced resistance to EAE in WT receptor mice (WT feces: WT mice with EAE and that received feces from WT mice, $n=5$; KO feces: WT mice with EAE and that received feces from CD44KO mice, $n=5$ ). (B) Results showing $L$. acidipiscis having induced resistance to EAE in WT mice (E. coli: WT mice with EAE and that were fed $E$. coli, $n=5$; L. a: WT mice with EAE and that were fed $L$. acidipiscis, $n=5$ ). (C) L. acidipiscis did not produce resistance to EAE in $\mathrm{TCR}^{-1-}$ mice (E. coli: $\mathrm{TCR}^{-1-}$ mice with EAE and that were fed $E$. coli, $n=5$; L. a: $\mathrm{TCR}^{-1-}$ mice with EAE and that were fed $L$. acidipiscis, $n=5$ ). This result suggested the presence of $\gamma \delta$ T cells to be required for realizing the protective effect of $L$. acidipiscis. Clinical scores were recorded daily after EAE induction and fecal transfer. The values are shown as mean \pm SEM. Data were analyzed using the Mann-Whitney $U$ test. ${ }^{\star \star} P<0.01,{ }^{\star \star *} P<0.001, \# P>0.05$.

day 15 post-immunization of mice with the $\mathrm{MOG}_{35-55}$ peptide, immunohistograms were acquired to detect the presence of $\gamma \delta \mathrm{T}$ cells (GL3 antibody staining positive) in the intestinal tissues of 
A

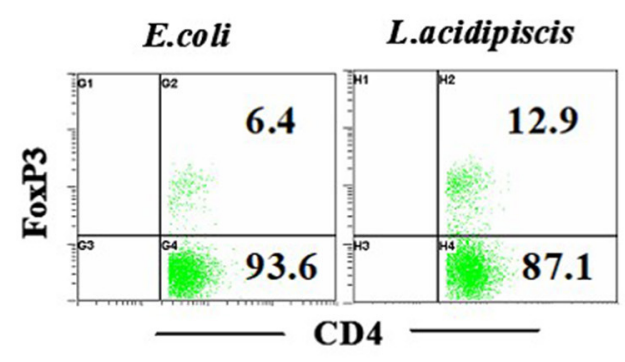

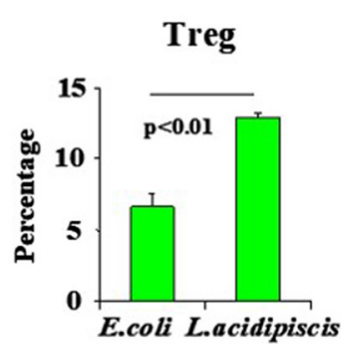

B

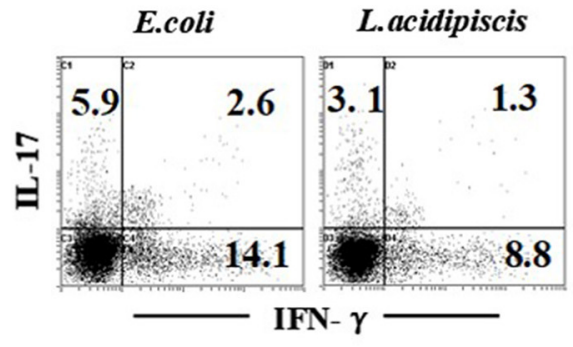

TH1

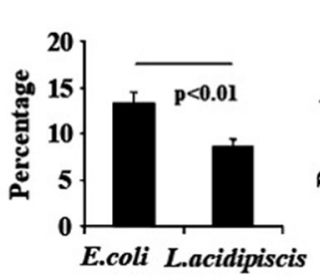

TH17

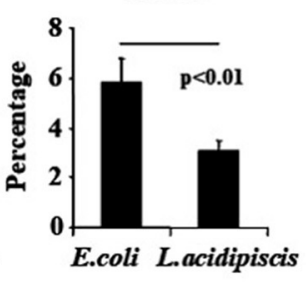

C
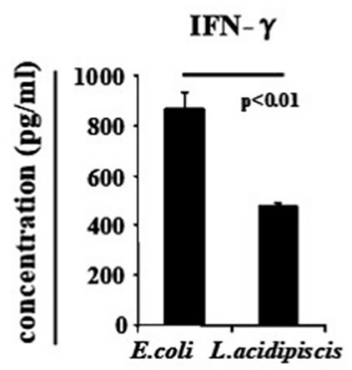

IL-17

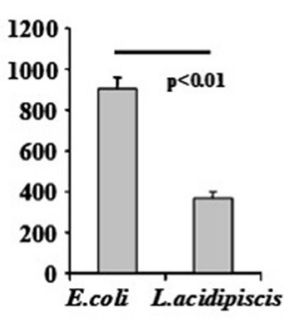

IL-10

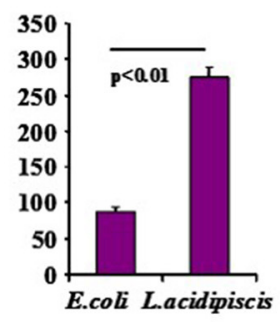

IL-13

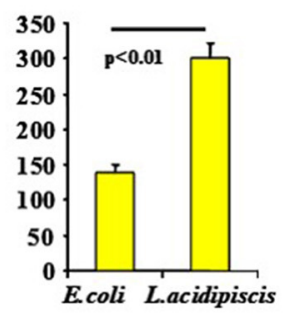

FIGURE 4 | L. acidipiscis induced the development of Treg cells and inhibited pathological differentiation into Th1 and Th17 cells. Percentages of CD4 ${ }^{+} \mathrm{T}$ cells consisting of Treg, Th1 and Th17 cells in mesenteric lymph nodes of C57BL/6 mice fed with bacteria were determined on day 15 when EAE symptoms peaked. (A) FACS detection of $\mathrm{CD}^{+}{ }^{+} \mathrm{FOXP3}^{+}$Treg cells, and percentage of $\mathrm{CD} 4^{+} \mathrm{T}$ cells consisting of Treg cells. (B) FACS detection of Th1 and Th17 cells, and percentage in $\mathrm{CD}^{+}+\mathrm{T}$ cells. (C) Cytokine concentration in cell culture supernatant. Representative flow cytometry plots were derived from a single experiment with 5 mice per experimental group and average percentage of each subset was expressed as mean $\pm \mathrm{SEM}$ from three independent experiments. Statistical differences were determined by using Student's $t$-test, and data with $P<0.01$ represent significant differences between the two groups.

EAE-resistant mice (CD44KO-EAE) and EAE-susceptible mice (WT-EAE) (arrow in Figure 5). Significantly more $\gamma \delta$ T cells were found in the intestinal tracts of CD $44 \mathrm{KO}$-EAE mice than in those of WT-EAE mice. We confirmed the resistance of CD44KO mice to EAE to be closely related to the presence of $\gamma \delta \mathrm{T}$ cells in the small intestinal epithelium of mice.

\section{L. acidipiscis Promoted the Development of $\mathrm{V}_{\gamma} 1^{+} \gamma \delta \mathrm{T}$ Cells and Inhibited $\mathrm{V}_{\gamma} 4^{+} \gamma \delta \mathrm{T}$ Cells in vitro}

To investigate the differential induction of $\mathrm{V} \gamma 1^{+/} \mathrm{V} \gamma 4^{+} \quad \gamma \delta$ $\mathrm{T}$ cells and $\mathrm{CD} 4^{+} \mathrm{Th}$ cells by $L$. acidipiscis, we examined the differentiation of $\gamma \delta \mathrm{T}$ cells and encephalitogenic $\mathrm{CD} 4^{+} \mathrm{T}$ cells from the splenocytes of EAE mice. $\gamma \delta \mathrm{T}$ and $\mathrm{CD}^{+} \mathrm{T}$ cells were co-cultured with or without L. acidipiscis strains for 3 days (Figure 6). The L. acidipiscis strain induced a significant increase in the proportion of $\mathrm{V} \gamma 1^{+} \gamma \delta \mathrm{T}$ cells, and it inhibited the proportion of $\mathrm{V}_{\gamma} 4^{+} \gamma \delta \mathrm{T}$ cells. In addition, the differentiation of Th2 cells was significantly enhanced after they were co-cultured with L. acidipiscis (Figure 6B), whereas the development of Th17 cells was significantly inhibited after they were co-cultured with L. acidipiscis (Figure 6C).

\section{DISCUSSION}

The cause of multiple sclerosis (MS) is unclear, and there are no effective methods for preventing and treating this disease. Immunology, genetics and histopathology data of patients with MS support the concept that autoimmunity plays a major role in the pathogenesis of the disease. Our current understanding of the pathogenesis of MS and that of its disease-causing mechanisms have mainly derived from the results of investigations using 
A

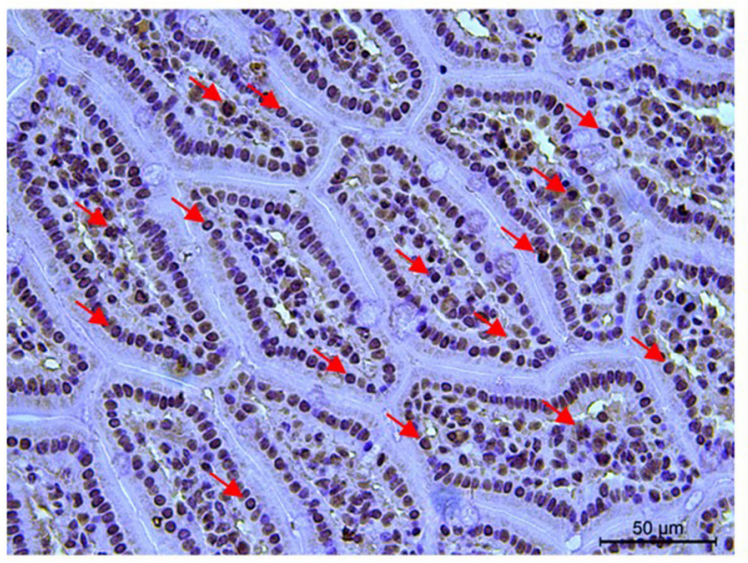

B

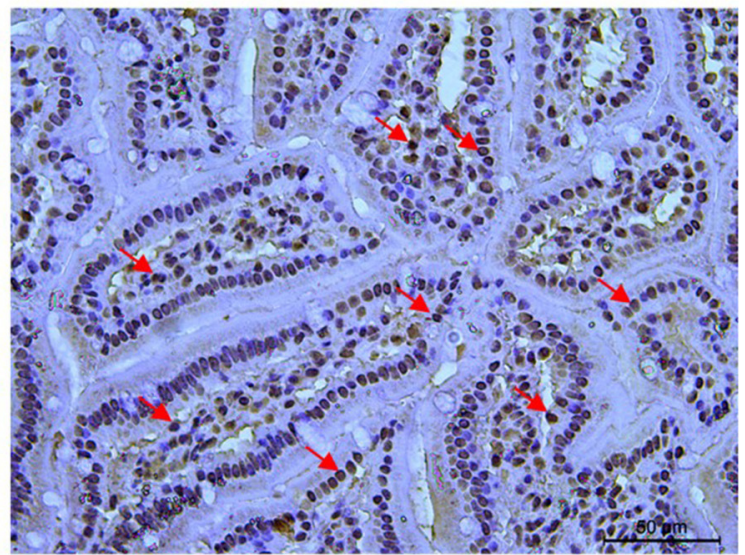

WT-EAE

$$
\begin{aligned}
& \text { WT-EAE } \\
& \text { KO-EAE }
\end{aligned}
$$

C

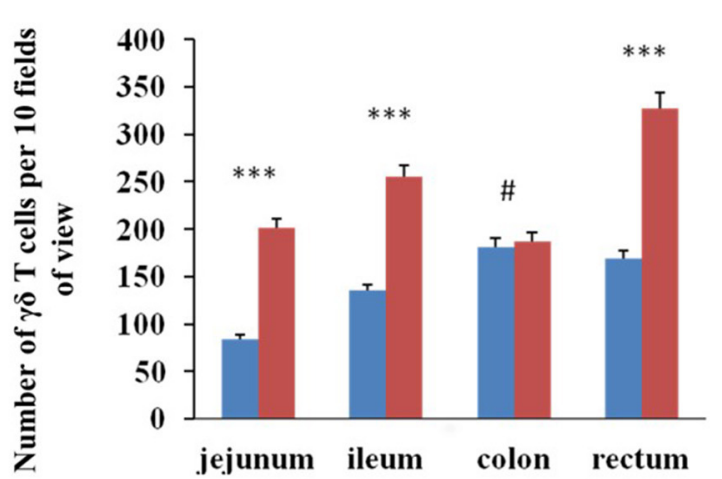

FIGURE 5 | Significantly more $\gamma \delta$ T cells were observed in the intestines of EAE-resistant mice. Representative histological appearances of jejunal tissues of CD44-EAE and WT-EAE mice on day 15 post-immunization, parts of $\gamma \delta$ T cells with GL3 positive staining were pointed with red arrows. (A) Jejunum of a CD44KO-EAE mice. (B) Jejunum of a WT-EAE mice. (C) Mean numbers of $\gamma \delta$ T cells in the jejunum, ileum, colon and rectum of 10 high-power field. Data from three separate experiments with five mice/group are presented as mean $\pm \mathrm{SEM}$. Data were analyzed with Student's $t$-test: ${ }^{\star \star \star} P<0.001, \# P>0.05$.

a classical mouse model; here, EAE is induced by performing subcutaneous immunization with an emulsion composed of a myelin component, such as MOG peptide, and complete Freund's adjuvant together with an administration of pertussis toxin. The activation of an autoimmune reaction and the production of myelin-specific $\mathrm{CD}^{+}{ }^{+} \mathrm{T}$ cells have been identified as being key to the development of MS. Therefore, inducing protective immune phenotype constitutes an important strategy for treatment of MS.

Highly heterogeneous microbial populations reside in the gastrointestinal tracts of mammals, and are essential for the immune systems of the hosts to completely develop. Intestinal microbes determine the development of the host microbial population and immune system, which are in a complex balance; the genetic material of such microbes has recently been coined as the "microbiome." Models for spontaneous EAE were found to be particularly useful for research on the role of gut microbiota in the induction of brain inflammation (22). The results of such research have indicated the signal communication of the gut bacteria-gut-brain response axis to be closely related to the occurrence of MS: when such animals are kept under germ-free conditions, no disease develops; but when their intestines are occupied by normal intestinal flora, disease is triggered. This model can be used to identify the bacterial components of intestinal flora, which can trigger and expand the pro-inflammatory $\mathrm{T}$ cells (Th1 and Th17 cells). Intestinal microorganisms come into contact with antigenpresenting cells (APCs) in the intestinal lumen or Peyer's patches. The APCs further present antigen to the naive $\mathrm{T}$ cells in the lamina propria. Naive $\mathrm{T}$ cells are further activated after migrating to mesenteric lymph nodes. The activated $\mathrm{T}$ cells diffuse through the blood and distal lymph nodes and migrate to the ileum propria (23). These results can help explain the mechanisms of chronic brain inflammation induced by antigen diffusion. Generally speaking, intestinal probiotics play an important role in MS, and can prevent and alleviate MS by inhibiting and treating inflammatory cells associated with this disease.

Gut-associated lymphoid tissue (GALT) is an important component of the immune system. Treg and Th17 cells induced 
A
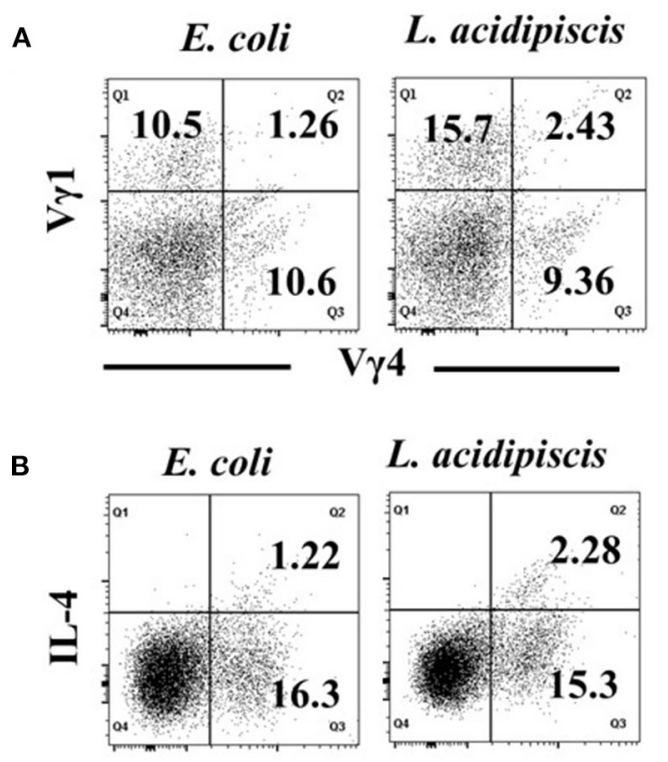

C

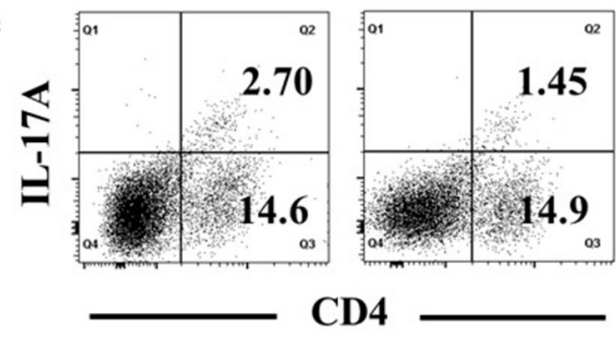

L. acidipiscis

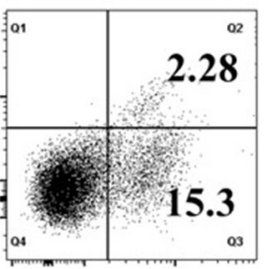

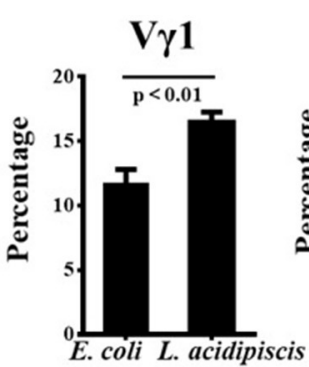
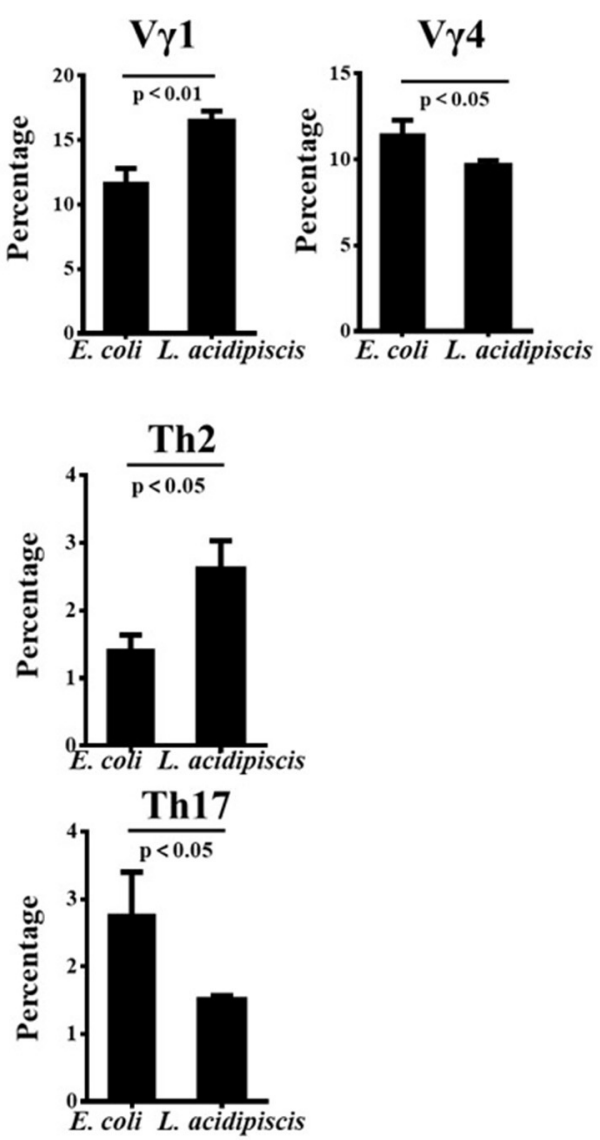

FIGURE 6 | L. acidipiscis promoted the development of $V \gamma 1^{+} \gamma \delta T$ cells and inhibited $V \gamma 4^{+} \gamma \delta T$ cells in vitro. (A) The development of $V \gamma 1^{+} \gamma \delta T$ cells was significantly enhanced while the development of $V \gamma 4^{+} \gamma \delta \mathrm{T}$ cells was significantly suppressed after they were co-cultured with $L$. acidipiscis. (B) The development of Th2 cells was significantly enhanced after they were co-cultured with L. acidipiscis. (C) The differentiation of Th17 cells was significantly inhibited after co-cultured with L. acidipiscis. Representative flow cytometry plots were derived from a single experiment with three mice per experimental group and average percentage of each subset was expressed as mean \pm SEM from three independent experiments. Data were analyzed with Student's $t$-test, and data with $P<0.01, P<0.05$ represent significant differences between the two groups.

by the intestinal tract are the characteristic $\mathrm{T}$ cells of the intestinal immune network (24). While IL-10 is responsible for maintaining the expression and function of FOXP3 in Treg cells (25). B. adolescentis IF1-03 has been shown to stimulate maturation of macrophages, producing higher levels of IL-10 and lower levels of IL- 6 and TGF- $\beta$, features consistent with the upregulation of Treg cells in DSS-colitis mice in vivo and splenocytes in vitro. On the other hand, B. adolescentis IF111 has been shown to stimulate macrophages to secrete higher levels of IL- 6 and TGF- $\beta$, and lower levels of IL-10, which promoted the differentiation of $\mathrm{CD}^{+}{ }^{+} \mathrm{T}$ cells into Th17 cells (26). Therefore, as far as health and disease are concerned, specific bacterial species have been proven to have a significant impact on the differentiation of immune cell subsets and innate immune maturation (27). Specifically, probiotics and prebiotics have been reported to have positive immuno-equilibrium restorative effects. Probiotics contribute to the balance of cytokines, and can positively influence the progress of allergic and inflammatory diseases. Our results showed that compared with E. coli, L. acidipiscis increased the production of $\mathrm{CD} 4^{+} \mathrm{FOXP}^{+}$Treg cells, IL-10 and IL-13, and inhibited the production of Th1, Th17, IFN- $\gamma$ and IL-17A. Clinical observations have shown beneficial clinical effects of probiotics, and probiotics strains may be used to treat inflammatory disease. Evidence has been presented to show that probiotics such as bifidobacterium and lactobacillus in the host can be involved in immune regulation by skewing of naive $\mathrm{T}$ cells toward Treg cells (28).

$\gamma \delta$ T cells appear earlier than do $\alpha \beta$ T cells in the development of thymus, mainly in the early stages of fetal development (29). Compared with $\alpha \beta \mathrm{T}$ cells, $\gamma \delta \mathrm{T}$ cells only represent a small number of $\mathrm{T}$ cell subsets $(1-10 \%)$ in the peripheral blood, and are mainly present in epithelial tissue in the form of intraepithelial lymphocytes (IELs). $\gamma \delta \mathrm{T}$ cells have their own distinct characteristics, such as relatively low TCR diversity and being able to directly recognize antigen without the requirement of a presentation of the antigen that are distinct from those of $\alpha \beta$ 
T cells (30). In addition, Benakis et al. demonstrated a reduction in ischemic brain damage in mice as a result of antibioticinduced changes in the intestinal flora, and that the effects could be transmitted through fecal transplantation. Furthermore, intestinal dysbiosis has been found to alter immune homeostasis in the small intestine, leading to an increase in the number of Treg cells and a reduction in IL-17-positive $\gamma \delta \mathrm{T}$ cells through altered dendritic cell (DC) activity (31). These studies have focused on the role of the intestinal flora and gut-brain axis and IL-17 (+) $\gamma \delta \mathrm{T}$ cells in MS and EAE as both pathogenic and protective, their role in the CNS, the types of subsets and a possible role in Th17 inflammation.

The heterogeneity of phenotype and function of $\gamma \delta \mathrm{T}$ cells is not clearly demonstrated so far. Studies have shown activated $\mathrm{V} \gamma 1^{+} \gamma \delta \mathrm{T}$ cells expressing relatively high levels of IL-4 and IL5 (32), and $\mathrm{V} \gamma 4^{+} \gamma \delta \mathrm{T}$ cells secreting relatively high amounts of IL-17A, IL-17F and IFN- $\gamma$ (33). $V \gamma 1^{+} \gamma \delta 17 \mathrm{~T}$ cells and $\mathrm{V} \gamma 4^{+} \gamma \delta 17 \mathrm{~T}$ cells are common subtypes of $\gamma \delta 17 \mathrm{~T}$ cells. Both cell types maintain the phenotype of producing IFN- $\gamma$, TNF$\alpha$, TGF- $\beta$ and IL-10. While $\mathrm{V} \gamma 1^{+} \gamma \delta \mathrm{T}$ cells produce more Th2-type cytokines such as IL-4 and IL-5, V $\gamma 4^{+} \gamma \delta \mathrm{T}$ cells preferentially produce IL-17 (34). These positive and negative effects may be related to the heterogeneity of $\gamma \delta$ T cells. Therefore, the induction of protective regulatory $\gamma \delta \mathrm{T}$ cells by intestinal probiotics constitutes a new strategy for treating MS. Overall, our results provided evidence that $L$. acidipiscis could influnce the differention of $\gamma \delta \mathrm{T}$ cells and $\mathrm{CD} 4^{+} \mathrm{T}$ cells into diffrent subsets in vitro, altough the data was limited and lack of in vivo evidence. The further study was needed in clarification of the related questions.

\section{CONCLUSIONS}

In this study, we found a negative correlation between $L$. acidipiscis in the intestinal tract and the progress of EAE. The resistance of CD44KO mice to EAE was related to the stimulation and activation of $\gamma \delta \mathrm{T}$ cells in small intestine epithelial tissues by $L$. acidipiscis. Here, we showed the presence of significantly more $\gamma \delta \mathrm{T}$ cells in the intestinal tracts of CD44KO-EAE mice than in those of WT-EAE mice. The resistance of CD44KO mice to EAE could be transmitted by intragastric administration of $L$. acidipiscis or feces transplanted from CD44KO mice. We analyzed the composition of intestinal flora and the levels of $L$. acidipiscis in EAE. Our results showed a negative correlation between the amount of intestinal $L$. acidipiscis

\section{REFERENCES}

1. Yamout BI, Alroughani R. Multiple sclerosis. Semin Neurol. (2018) 38:21225. doi: $10.1055 / \mathrm{s}-0038-1649502$

2. Mowry EM, Glenn JD. The dynamics of the gut microbiome in multiple sclerosis in relation to disease. Neurol Clin. (2018) 36:18596. doi: 10.1016/j.ncl.2017.08.008

3. Gran B, Rostami A. T cells, cytokines, and autoantigens in multiple sclerosis. Curr Neurol Neurosci Rep. (2001) 1:263-70. doi: 10.1007/s11910001-0029-3 and the progression of EAE, and showed the regulation of encephalitogenic $\mathrm{CD}^{+} \mathrm{T}$ cell differentiation by L. acidipiscis to be related to $\gamma \delta \mathrm{T}$ cells. Meanwhile in our experiments, $L$. acidipiscis suppressed in vitro the proliferation of Th1 and Th17 cells as well as the secretion of IFN- $\gamma$ and IL-17A, and clearly promoted the development of Treg and Th2 cells. In contrast, EAE was not significantly inhibited when $L$. acidipiscis was fed to $\mathrm{TCR} \delta^{-/-}$mice. In summary, we have provided evidence for L. acidipiscis being a critical factor in the development of mouse Treg cells. In our experiments, L. acidipiscis was used to induce regulatory $\mathrm{T}$ cells to differentiate into protective cell subsets, thus EAE-susceptible mice obtaining the resistance to EAE. In addition, our results demonstrated an association between progression of MS and a decreased proportion of $L$. acidipiscis in the host intestinal tract, and demonstrated the ability of $L$. acidipiscis to target ${\mathrm{V} \gamma 1^{+}}^{+}$and $\mathrm{V} \gamma 4^{+} \gamma \delta \mathrm{T}$ cells and to interfere with the expression of IL-10, IL-13, IFN- $\gamma$ and IL-17A.

\section{DATA AVAILABILITY STATEMENT}

The raw data supporting the conclusions of this article will be made available by the authors, without undue reservation.

\section{ETHICS STATEMENT}

The animal study was reviewed and approved by Institutional Animal Care and Use Committee of Guangzhou Medical University.

\section{AUTHOR CONTRIBUTIONS}

HG contributed to conception and design of the study. SR and $\mathrm{XZ}$ performed the experiments. $\mathrm{LW}$ and $\mathrm{DH}$ carried out data analysis. All authors participated in drafting of the manuscript and critical revision of the draft and contributed to the article and approved the submitted version.

\section{FUNDING}

We gratefully acknowledge the financial support from the Guangzhou Science, Technology and Innovation Commission (grant number: 201707010026), Guangdong Science and Technology Department (grant number: 2017A020215141), and Guangzhou Municipal Health and Family Planning Commission (grant number: 20181A011101). 
7. Chitrala KN, Guan H, Singh NP, Busbee B, Gandy A, Mehrpouya-Bahrami $\mathrm{P}$, et al. CD44 deletion leading to attenuation of experimental autoimmune encephalomyelitis results from alterations in gut microbiome in mice. Eur J Immunol. (2017) 47:1188-99. doi: 10.1002/eji.201646792

8. Jin C, Lagoudas GK, Zhao C, Bullman S, Bhutkar A, Hu B, et al. Commensal microbiota promote lung cancer development via $\gamma \delta \mathrm{T}$ cells. Cell. (2019) 176:998-1013.e16. doi: 10.1016/j.cell.2018.12.040

9. Ogata M, Itoh T. Gamma/delta intraepithelial lymphocytes in the mouse small intestine. Anat Sci Int. (2016) 91:301-12. doi: 10.1007/s12565-016-0341-2

10. McKenzie DR, Kara EE, Bastow CR, Tyllis TS, Fenix KA, Gregor CE, et al. IL-17-producing $\gamma \delta \mathrm{T}$ cells switch migratory patterns between resting and activated states. Nat Commun. (2017) 8:15632. doi: 10.1038/ncomms15632

11. Papotto PH, Reinhardt A, Prinz I, Silva-Santos B. Innately versatile: $\gamma \delta 17 \mathrm{~T}$ cells in inflammatory and autoimmune diseases. J Autoimmun. (2018) 87:2637. doi: 10.1016/j.jaut.2017.11.006

12. Welte T, Lamb J, Anderson JF, Born WK, O’Brien RL, Wang T. Role of two distinct $\gamma \delta \mathrm{T}$ cell subsets during West Nile virus infection. FEMS Immunol Med Microbiol. (2008) 53:275-83. doi: 10.1111/j.1574-695X.2008.00430.x

13. Dong P, Zhang S, Cai M, Kang N, Hu Y, Cui L, et al. Global characterization of differential gene expression profiles in mouse $\mathrm{V} \gamma 1+$ and $\mathrm{V} \gamma 4+\gamma \delta \mathrm{T}$ cells. PLoS ONE. (2014) 9:e112964. doi: 10.1371/journal.pone.0112964

14. Casanova MR, Azevedo-Silva J, Rodrigues LR, Preto A. Colorectal cancer cells increase the production of short chain fatty acids by propionibacterium freudenreichii impacting on cancer cells survival. Front Nutr. (2018) 5:44. doi: 10.3389/fnut.2018.00044

15. Stanisavljevic S, Lukic J, Momcilovic M, Miljkovic M, Jevtic B, Kojic M, et al. Gut-associated lymphoid tissue, gut microbes and susceptibility to experimental autoimmune encephalomyelitis. Benef Microbes. (2016) 7:36373. doi: 10.3920/BM2015.0159

16. Guan H, Nagarkatti PS, Nagarkatti M. CD44 reciprocally regulates the differentiation of encephalitogenic Th1/Th17 and Th2/regulatory $\mathrm{T}$ cells through epigenetic modulation involving DNA methylation of cytokine gene promoters, thereby controlling the development of experimental autoimmune encephalomyelitis. J Immunol. (2011) 186:6955-64. doi: 10.4049/jimmunol.1004043

17. Caporaso JG, Lauber CL, Walters WA, Berg-Lyons D, Huntley J, Fierer N, et al. Ultra-high-throughput microbial community analysis on the Illumina HiSeq and MiSeq platforms. ISME J. (2012) 6:16214. doi: 10.1038/ismej.2012.8

18. Zhao G, Nyman M, Jonsson JA. Rapid determination of short-chain fatty acids in colonic contents and faeces of humans and rats by acidified waterextraction and direct-injection gas chromatography. Biomed Chromatogr. (2006) 20:674-82. doi: 10.1002/bmc.580

19. Muller PA, Koscso B, Rajani GM, Stevanovic K, Berres ML, Hashimoto D, et al. Crosstalk between muscularis macrophages and enteric neurons regulates gastrointestinal motility. Cell. (2014) 158:300-13. doi: 10.1016/j.cell.2014.04.050

20. Guan H, Fan D, Mrelashvili D, Hao H, Singh NP, Singh UP, et al. MicroRNA let-7e is associated with the pathogenesis of experimental autoimmune encephalomyelitis. Eur J Immunol. (2013) 43:104-14. doi: 10.1002/eji.201242702

21. Kang N, Tang L, Li X, Wu D, Li W, Chen X, et al. Identification and characterization of Foxp3(+) gammadelta $\mathrm{T}$ cells in mouse and human. Immunol Lett. (2009) 125:105-13. doi: 10.1016/j.imlet.2009.06.005
22. Berer K, Mues M, Koutrolos M, Rasbi ZA, Boziki M, Johner C, et al. Commensal microbiota and myelin autoantigen cooperate to trigger autoimmune demyelination. Nature. (2011) 479:53841. doi: 10.1038/nature10554

23. Ochoa-Repáraz J, Mielcarz DW, Begum-Haque S, Kasper LH. Gut, bugs, and brain: role of commensal bacteria in the control of central nervous system disease. Ann Neurol. (2011) 69:240-7. doi: 10.1002/ana.22344

24. Atarashi K, Umesaki Y, Honda K. Microbiotal influence on $\mathrm{T}$ cell subset development. Semin Immunol. (2011) 23:14653. doi: 10.1016/j.smim.2011.01.010

25. Neumann C, Scheffold A, Rutz S. Functions and regulation of $\mathrm{T}$ cell-derived interleukin-10. Semin Immunol. (2019) 44:101344. doi: 10.1016/j.smim.2019.101344

26. Yu R, Zuo F, Ma H, Chen S. Exopolysaccharide-Producing Bifidobacterium adolescentis strains with similar adhesion property induce differential regulation of inflammatory immune response in Treg/Th17 Axis of DSScolitis mice. Nutrients. (2019) 11:782. doi: 10.3390/nu11040782

27. Rooks MG, Garrett WS. Gut microbiota, metabolites and host immunity. Nat Rev Immunol. (2016) 16:341-52. doi: 10.1038/nri.2016.42

28. Laiño J, Villena J, Kanmani P, Kitazawa H. Immunoregulatory effects triggered by lactic acid bacteria exopolysaccharides: new insights into molecular interactions with host cells. Microorganisms. (2016) 4:27. doi: 10.3390/microorganisms 4030027

29. Bonneville M, O’Brien RL, Born WK. $\gamma \delta \mathrm{T}$ cell effector functions: a blend of innate programming and acquired plasticity. Nat Rev Immunol. (2010) 10:467-78. doi: 10.1038/nri2781

30. Brenner MB, McLean J, Dialynas DP, Strominger JL, Smith JA, Owen FL, et al. Pillars article: identification of a putative second T-cell receptor. Nature. 1986. 322: 145-149. J Immunol. (2016) 196:3509-13. doi: 10.1038/322145a0

31. Benakis C, Brea D, Caballero S, Faraco G, Moore J, Murphy M, et al. Commensal microbiota affects ischemic stroke outcome by regulating intestinal $\gamma \delta$ T cells. Nat Med. (2016) 22:516-23. doi: 10.1038/nm.4068

32. Wehrmann F, Lavelle JC, Collins CB, Tinega AN, Thurman JM, Burnham EL, et al. $\gamma \delta$ T cells protect against LPS-induced lung injury. J Leukoc Biol. (2016) 99:373-86. doi: 10.1189/jlb.4A0115-017RR

33. Sun G, Yang S, Cao G, Wang Q, Hao J, Wen Q, et al. $\gamma \delta$ T cells provide the early source of IFN-gamma to aggravate lesions in spinal cord injury. $J$ Exp Med. (2018) 215:521-35. doi: 10.1084/jem.20170686

34. Blink SE, Caldis MW, Goings GE, Harp CT, Malissen B, Prinz $\mathrm{I}$, et al. $\gamma \delta \mathrm{T}$ cell subsets play opposing roles in regulating experimental autoimmune encephalomyelitis. Cell Immunol. (2014) 290:39-51. doi: 10.1016/j.cellimm.2014.04.013

Conflict of Interest: The authors declare that the research was conducted in the absence of any commercial or financial relationships that could be construed as a potential conflict of interest.

Copyright (c) 2021 Ren, Zhang, Guan, Wu, Yu, Hou, Yan and Fang. This is an open-access article distributed under the terms of the Creative Commons Attribution License (CC BY). The use, distribution or reproduction in other forums is permitted, provided the original author(s) and the copyright owner(s) are credited and that the original publication in this journal is cited, in accordance with accepted academic practice. No use, distribution or reproduction is permitted which does not comply with these terms. 